challenging epithelial and subepithelial neoplasms that are not amenable to conventional resection techniques.

Methods This was a retrospective case series study of patients underwent two methods of endoscopic full-thickness resection (EFTR), either of which was deep resection using ESD knives and post-resection closure with OTSC (Group 1), the other was pre-resection closure with OTSC and second EFTR with snare (Group 2).

Results Of all 21 patients, 11 cases were in Group 1 and 10 in Group 2. The mean time of EFTR procedure was 76.83 $\pm 34.97 \mathrm{~min}$ in Group 1 which was significantly longer than that of Group $2(p=0.0128)$. The mean time of OTSC closure and length of hospital stay of Group 1 were also longer compared to Group 2, but the difference was not significant. Both of complete resection (R0) and technical success rate of Group 1 were 83.3\% and were both $100 \%$ for Group 2.

Conclusions EFTR for pre-resection closure is potentially faster compared with the concept of applying closure after EFTR. Larger prospective controlled studies comparing those two techniques are warranted in the future.

\section{IDDF2018-ABS-0102 RELATIONSHIP BETWEEN HISTOLOGICAL CHARACTERISTICS AND BRAF MUTATION, P53, KI67 IMMUNOEXPRESSION IN PATIENTS WITH COLORECTAL POLYP IN THAI NGUYEN}

Luu Thi Binh*, Doan Anh Thang, Nguyen Trong Hieu, Tran Duc Quy, Dong Duc Hoang. Thai Nguyen University of Medicine and Pharmacy, Vietnam

\subsection{6/gutjnl-2018-IDDFabstracts. 110}

Background Changes in immune expression of BRAF, P53, and Ki67 proteins are part of the colorectal carcinogenic mechanisms associated with colorectal polyps.

Aims To analyse the relationship between histological characteristics and BRAF mutation, P53, Ki67 immunoexpression in patients with a colorectal polyp.

Methods A total of 81 non-cancerous colorectal polyp patients were randomised to the study. Patients undergoing endoscopic, histopathological and immunohistochemical analyses.

Results BRAF mutation in the dysplasia area showed an average of $1 \pm 0.69$, non-dysplasia $0.33 \pm 0.49, \quad \mathrm{p}=0.001 ; \quad$ P53 expression in dysplasia was found to be $1.74 \pm 1.38$, non-dysplasia $1.22 \pm 1.05, p=0.001$. Ki67 expression in dysplasia was found to be $1.33 \pm 1.18$, non-dysplasia $1.20 \pm 0.97, \mathrm{p}=0.002$. The BRAF mutation in dysplasia area detected $50 \%$ of the traditional serrated polyps. BRAF mutation in dysplasia had $25 \%$ level ++ , in pervasive samples, $\mathrm{p}<0.001$. P53 expression in dysplasia was $68.6 \%$ level +++ in adenoma polyps group, in non-dysplasia area rate $33.3 \%$ level +++ in non-neoplastic polyp, $\mathrm{p}<0.001$. P53 expression were mainly found in the bottom samples, the dysplasia area was $44.3 \%$ level +++ , non-dysplasia $15.7 \%$ level,$+++ p=0.02$. Ki67 expression in dysplasia area was $35.3 \%$ in the level of +++ adenoma polyps, non-dysplasia at $20 \%$ level +++ non neoplastic polyps, $\mathrm{p}<0.001$. Ki67 expression of dysplasia $28.6 \%$ level +++ was mainly found in the bottom samples, $\mathrm{p}<0.001$.

Conclusions BRAF mutation is more common in serrated polyps, P53, Ki67 expression are more common in adenomatous polyps. Immunohistochemical changes of these proteins are more common in the dysplasia area and there are differences between cell layers.

\section{IDDF2018-ABS-0103 RELATIONSHIP BETWEEN ENDOSCOPIC CHARACTERISTICS AND BRAF MUTATION, P53, KI67 IMMUNOEXPRESSION IN PATIENTS WITH COLORECTAL POLYP IN THAI NGUYEN}

Luu Thi Binh*, Doan Anh Thang, Nguyen Trong Hieu, Tran Duc Quy, Dong Duc Hoang. Thai Nguyen University of Medicine and Pharmacy, Vietnam

\subsection{6/gutjnl-2018-IDDFabstracts.111}

Background Co-results of endoscopy and BRAF mutation, P53, Ki67 immunoexpression increase sensitivity and specificity in the early diagnosis of colorectal cancer.

Aims To analyse the relationship between endoscopic characteristics and BRAF mutation, P53, Ki67 immunoexpression in patients with a colorectal polyp.

Methods A total of 81 non-cancerous colorectal polyp patients were randomised to the study. Patients undergoing endoscopic, histopathological, and immunohistochemical analyses.

Results Gender ratio: male 69.1\%, female 30.9\%. Highest age group 41-60 was 58\%. There are a total of 175 polyps, an average number per patient $2.16 \pm 1.14$. The proportion of multiple polyp patients was $61.7 \%$, single polyp $38.3 \%$. Sessile polyp was highest: $38.3 \%$. The size of polyps $<1 \mathrm{~cm}$ was the highest rate, with $46.9 \%,>2 \mathrm{~cm}$ with only $7.4 \%$. Colon polyps accounted for $67.9 \%$, rectal $32.1 \%$. BRAF mutation in single polyp accounted for $22.8 \%$, multiple polyps $22 \%$. BRAF mutation in flat-elevated polyp were lowest with $20 \%$, pedunculated polyps had the highest rate of $26.9 \%$. Mutation of BRAF polyp size $>2 \mathrm{~cm}$ accounted for $50 \%$ as the highest. P53 expression was similar in the single and multiple polyp. P53 expression level +++ in pedunculated polyp with $57.7 \%$, subpedunculated $57.1 \%$, sessile $32.3 \%$. In terms of size, $100 \%$ polyps $>2 \mathrm{~cm}$ express P53, p=0.03. Ki67 expression was similar in the single and multiple polyp. Polyp size $>2 \mathrm{~cm}$ accounted for the highest Ki67 expression rate of $66.6 \%$. Pedunculated polyp had the highest rate of Ki67 expression in the dysplasia of level +++ with $42.3 \%$, subpedunculated and sessile polyp $16.1 \%, \mathrm{p}=0.04$.

Conclusions P53 immunoexpression are usually manifested in large pedunculated and subpedunculated polyps. Ki67 immunoexpression in the dysplasia area, manifested in pedunculated and subpedunculated polyps.

\section{IDDF2018-ABS-0115 THE ASSOCIATION BETWEEN ELECTRONIC CIGARETTE SMOKING AND GASTROESOPHAGEAL REFLUX DISEASE IN ADULT URBAN POPULATION}

${ }^{1}$ Ahmad Fariz Malvi Zamzam Zein*, ${ }^{2}$ Catur Setiya Sulistiyana, ${ }^{3}$ Murdani Abdullah. ${ }^{1}$ Department of Internal Medicine, Faculty of Medicine, Swadaya Gunung Jati University, Indonesia; ${ }^{2}$ Department of Medical Education, Faculty of Medicine, Swadaya Gunung Jati University, Indonesia; ${ }^{3}$ Division of Gastroenterology, Department of Internal Medicine, Cipto Mangunkusumo Hospital, Indonesia

\subsection{6/gutjnl-2018-IDDFabstracts. 112}

Background This study was aimed to investigate the association between electronic cigarette (e-cig) smoking and gastroesophageal reflux disease (GERD) among the adult urban population.

Methods A cross-sectional study was conducted among 267 adult people in Cirebon City, West Java, Indonesia. A selfadministered questionnaire based on earlier validated GERD 